\title{
NOTE SUPPLEMENTARY TO THE PAPER "ON CERTAIN PAIRS \\ OF TRANSCENDENTAL FUNCTIONS WHOSE ROOTS
}

\author{
SEPARATE EACH OTHER"
}

BY

\section{MAXIME BÔCHER}

In the paper with the above title, which appeared in these $\mathrm{T} \mathrm{r}$ a $\mathrm{s}$ a c $\mathrm{t}$ i o n s, vol. 2 (1901), p. 428, I obtained a number of general theorems concerning the zeros of functions of the form $\phi_{2} y^{\prime}-\phi_{1} y$, where $y$ is a solution of a homogeneous linear differential equation of the second order. There $s$ a further general theorem of the same character which I should have included in that paper if I had discovered it at the time, and which I now give as VII' below, it being a counterpart to VII in the earlier paper. I also give three special applications of this general result, numbered VIII', VIII", VIII'", since they are counterparts of VIII. A part of VIII' was obtained by Fite in the Annals of Mathematics for June, 1917, (see the closing lines of his article) by another method; and it was this special result of Fite's that suggested to me the much more general Theorem VII'.

What follows should be regarded as an addendum to the former paper, to be inserted on page 434 at the end of $\S 2$. All references are to that paper, to which the reader should turn for an explanation of the notation and a statement of the restrictions placed on the functions.

$\mathrm{VII}^{\prime}$. If none of the six functions

$\phi_{1} \psi_{2}-\phi_{2} \psi_{1}, \psi_{1} \chi_{2}-\psi_{2} \chi_{1}, \chi_{1} \phi_{2}-\chi_{2} \phi_{1},\left\{\phi_{1}, \phi_{2}\right\},\left\{\psi_{1}, \psi_{2}\right\},\left\{\chi_{1}, \chi_{2}\right\}$ vanishes in (I), and if the last of them has the opposite sign from the two preceding ones, then no one of the functions $\Phi, \Psi, \mathrm{X}$ can vanish more than once in (I): and no two of them can vanish except perhaps $\Phi$ and $\Psi$. If these last two functions do both vanish in (I), the root of $\Phi$ will be greater or less than the root of $\Psi$ according as the product of the six functions written above is negative or positive.

The first part of this theorem is an immediate consequence of IV, p. 431. Let us then assume that $\Phi$ vanishes at $x_{1}, \Psi$ at $x_{2}$. These two points cannot coincide, since then $\phi_{1} \psi_{2}-\phi_{2} \psi_{1}$ would vanish at this point. 
Suppose, first, that $x_{1}<x_{2}$. Then

$$
\operatorname{sgn} \Phi\left(x_{2}\right)=\operatorname{sgn} \Phi^{\prime}\left(x_{1}\right),
$$

and, consequently, by (3), p. 430 ,

$$
\operatorname{sgn} \Phi\left(x_{2}\right)=-\operatorname{sgn}\left(\phi_{1} \psi_{2}-\phi_{2} \psi_{1}\right) \operatorname{sgn}\left\{\phi_{1}, \phi_{2}\right\} \operatorname{sgn} \Psi\left(x_{1}\right) .
$$

On the other hand, if we substitute in the identity (6), p. 432, first $x=x_{1}$, then $x=x_{2}$, we find

$$
\begin{aligned}
& \operatorname{sgn}\left(\phi_{1} \psi_{2}-\phi_{2} \psi_{1}\right) \operatorname{sgn} X\left(x_{1}\right)=-\operatorname{sgn}\left(\chi_{1} \phi_{2}-\chi_{2} \phi_{1}\right) \operatorname{sgn} \Psi\left(x_{1}\right), \\
& \operatorname{sgn}\left(\phi_{1} \psi_{2}-\phi_{2} \psi_{1}\right) \operatorname{sgn} X\left(x_{2}\right)=-\operatorname{sgn}\left(\psi_{1} \chi_{2}-\psi_{2} \chi_{1}\right) \operatorname{sgn} \Phi\left(x_{2}\right) .
\end{aligned}
$$

Multiplying together the last three equations, and remembering that $\mathbf{X}$ does not vanish, so that

we find

$$
\operatorname{sgn} X\left(x_{1}\right)=\operatorname{sgn} X\left(x_{2}\right)= \pm 1,
$$

$\operatorname{sgn} \Phi\left(x_{2}\right)=-\operatorname{sgn}\left[\left(\phi_{1} \psi_{2}-\phi_{2} \psi_{1}\right)\left(\chi_{1} \phi_{2}-\chi_{2} \phi_{1}\right)\left(\psi_{1} \chi_{2}-\psi_{2} \chi_{1}\right)\right]$

$$
\operatorname{sgn}\left\{\phi_{1}, \phi_{2}\right\} \operatorname{sgn} \Phi\left(x_{2}\right) \text {. }
$$

If here we cancel the factor $\operatorname{sgn} \Phi\left(x_{2}\right)= \pm 1$ from both sides, and replace -1 by its value sgn $\left\{\psi_{1}, \psi_{2}\right\}$ sgn $\left\{\chi_{1}, \chi_{2}\right\}$, we infer from this equation that the product of the six functions of $\mathrm{VII}^{\prime}$ is positive.

In a precisely similar manner, we show that if $x_{1}>x_{2}$, the product of these six factors is negative, and this completes the proof of $\mathrm{VII}^{\prime}$.

The theorem just proved admits of extensions in two directions. A brief mention of these will be sufficient.

First, the assumption that $\left\{\phi_{1}, \phi_{2}\right\},\left\{\psi_{1}, \psi_{2}\right\},\left\{\chi_{1}, \chi_{2}\right\}$ do not vanish may be replaced (at least if we assume that $\phi_{1}, \phi_{2}, \psi_{1}, \psi_{2}, \chi_{1}, \chi_{2}$ have continuous first derivatives) by the less restrictive conditions that these three functions do not change sign and that none of them vanishes at all points of a connected subinterval of $(\mathrm{I})$. This extension is effected as in similar cases on p. 436 .

Secondly, we may suppose that $\Phi$, instead of being of the form $\phi_{2} y^{\prime}-\phi_{1} y$, is of the form $\phi_{1} y-\phi_{2} z$, where $(y, z)$ is a solution of a pair of homogeneous linear differential equations of the first order. Making corresponding changes in the definitions of $\Psi$ and $X$, we obtain in this way a counterpart to XVI in these Transactions, vol. 3 (1902), p. 207, the proof being closely parallel to that we have here given.

We make a first application of $\mathrm{VII}^{\prime}$ by letting

$$
\Phi=y, \quad \Psi=y^{\prime}, \quad \mathbf{X}=p y^{\prime}+q y=-y^{\prime \prime},
$$


so that

$$
\begin{aligned}
& \phi_{1} \psi_{2}-\phi_{2} \psi_{1}=-1, \quad \psi_{1} \chi_{2}-\psi_{2} \chi_{1}=q, \quad \chi_{1} \phi_{2}-\chi_{2} \phi_{1}=p, \\
& \left\{\phi_{1}, \phi_{2}\right\}=1, \quad\left\{\psi_{1}, \psi_{2}\right\}=q, \quad\left\{\chi_{1}, \chi_{2}\right\}=q^{2}\left[\frac{d}{d x}\left(\frac{p}{q}\right)+1\right] .
\end{aligned}
$$

Theorem VII' now becomes*

VIII'. If

$$
q>0, \quad \frac{d}{d x}\left(\frac{p}{q}\right)<-1, \quad p \text { nowhere zero, }
$$

then no one of the functions $y, y^{\prime}, y^{\prime \prime}$ can vanish more than once; and no two of them can vanish except $y$ and $y^{\prime}$. If these two functions do both vanish, the root of $y$ is greater or less than the root of $y^{\prime}$ according as $p$ is negative or positive.

As a second application of $\mathrm{VII}^{\prime}$, we let

and thus find

$$
\mathrm{X}=y, \quad \Psi=y^{\prime}, \quad \Phi=-y^{\prime \prime}
$$

VIII". If

$$
q<0, \quad \frac{d}{d x}\left(\frac{p}{q}\right)<-1, \quad p \text { nowhere zero, }
$$

then no one of the functions $y, y^{\prime}, y^{\prime \prime}$ can vanish more than once; and no two of them can vanish except $y^{\prime}$ and $y^{\prime \prime}$. If these two functions do both vanish, the root of $y^{\prime}$ is greater or less than the root of $y^{\prime \prime}$ according as $p$ is negative or positive.

Finally, letting

we obtain

$$
\Phi=y, \quad \mathrm{X}=y^{\prime}, \quad \Psi=-y^{\prime \prime},
$$

VIII'"'. If

$$
q<0, \quad \frac{d}{d x}\left(\frac{p}{q}\right)>-1, \quad p \text { nowhere zero, }
$$

then no one of the functions $y, y^{\prime}, y^{\prime \prime}$ can vanish more than once; and no two of them can vanish except $y$ and $y^{\prime \prime}$. If these two functions do both vanish, the root of $y$ is greater or less than the root of $y^{\prime \prime}$ according as $p$ is negative or positive.

HaRVARD UNIVERSITY

July, 1917

* The latter part of this theorem is the result of Fite above referred to, except that Fite requires merely

$$
\frac{d}{d x}\left(\frac{p}{q}\right) \leqq-1
$$

This generalization, however, is not a substantial one, since the equality sign may be removed by an arbitrarily small variation of $p$, and such a variation, if the root of $y^{\prime}$ is held fixed, produces only a slight change in the root of $y$. 